PROCEEDINGS OF THE

AMERICAN MATHEMATICAL SOCIETY

Volume 133, Number 8, Pages 2273-2282

S 0002-9939(05)07949-9

Article electronically published on March 14, 2005

\title{
SPACE-TIME FRACTIONAL DERIVATIVE OPERATORS
}

\author{
BORIS BAEUMER, MARK M. MEERSCHAERT, AND JEFF MORTENSEN
}

(Communicated by Jonathan M. Borwein)

\begin{abstract}
Evolution equations for anomalous diffusion employ fractional derivatives in space and time. Linkage between the space-time variables leads to a new type of fractional derivative operator. This paper develops the mathematical foundations of those operators.
\end{abstract}

\section{INTRODUCTION}

In classical diffusion, particles spread in a normal bell-shaped pattern whose width grows like the square root of time. Anomalous diffusion occurs when the growth rate or the shape of the particle distribution is different than the classical model predicts. Anomalous diffusion is observed in many physical situations, motivating the development of new mathematical and physical models [5, 6] 7] [3] 16, 20. Some of the most successful models employ fractional derivatives [21, 27. in place of the usual integer order derivatives in the diffusion equation. One way to develop physically meaningful models for anomalous diffusion is to derive the limiting distribution of an ensemble of particles following a specified stochastic process. Continuous time random walks [22, 29, where each random particle jump occurs after a random waiting time, can be used to derive these limits [18, 20, 30]. Very large particle jumps are associated with fractional derivatives in space [14, while very long waiting times lead to fractional derivatives in time [18, 26]. The same model equations have also been applied to chaotic dynamics [31] and finance 10, 28, where empirical evidence [24, 25] shows that the waiting time between transactions is correlated with the ensuing price jump.

In the continuous time random walk, the size of the particle jumps can depend on the waiting time between jumps. For these models, the limiting particle distribution is governed by a fractional differential equation involving coupled space-time fractional derivative operators [3, 19]. This paper develops the mathematical foundations of those operators. In particular, they are shown to be the generators of certain continuous convolution semigroups, and their domain is identified. The

Received by the editors April 25, 2003.

2000 Mathematics Subject Classification. Primary 47G30; Secondary 60J60.

Key words and phrases. Evolution equation, anomalous diffusion, fractional derivative.

The first author was partially supported by the Marsden fund, administered by the Royal Society of New Zealand.

The second author was partially supported by NSF grants DMS-0139927 and DMS-0417869 as well as the Marsden fund, administered by the Royal Society of New Zealand. 
general form of the operator in ordinary space-time is also provided. The technical tools used in this development are semigroups of operators [1, 11, 23], and the theory of operator stable probability distributions [12, 15].

\section{Fractional Derivatives AND ANOMAlous Diffusion}

Let $C(x, t)$ denote the relative concentration of particles at location $x \in \mathbb{R}$ at time $t$. The classical diffusion equation $\partial_{t} C=\frac{1}{2} \partial_{x}^{2} C$ can be solved using the Fourier transform $c(k, t)=\int e^{i k x} C(x, t) d x$, which converts the diffusion equation to an ordinary differential equation $d c / d t=\frac{1}{2}(-i k)^{2} c$. The initial condition $c(k, 0) \equiv 1$ is equivalent to $C(x, 0)=\delta(x)$, so that all particles start at position $x=0$ at time $t=0$. The solution $c(k, t)=\exp \left(-\frac{1}{2} k^{2} t\right)$ inverts to a normal probability density with mean zero and standard deviation $\sqrt{t}$. This is also, using the central limit theorem, the limiting density of a random walk of particle jumps when the jumps have mean zero and variance one; and hence the standard Brownian Motion model.

If the probability distribution of the particle jumps has symmetric regularly varying tails with index $-\alpha$ for some $0<\alpha<2$ (roughly speaking, this means that the probability of jumping a distance greater than $r$ falls off like $r^{-\alpha}$ ), then the variance is undefined, so the classical central limit theorem does not apply. An extended central limit theorem [8, 9, 15] implies that the random walk converges to a stable Lévy motion whose probability density $C(x, t)$ has Fourier transform $c(k, t)=\exp \left(-|k|^{\alpha} t\right)$, evidently the solution to $d c / d t=-|k|^{\alpha} c$ with $c(k, 0) \equiv 1$. Inverting shows that the particle concentration solves a fractional partial differential equation $\partial_{t} C=\partial_{|x|}^{\alpha} C$, where the symmetric fractional derivative operator $\partial_{|x|}^{\alpha}$ corresponds to multiplication by the symbol $-|k|^{\alpha}$ in Fourier space. This is the usual fractional power of the second derivative operator. Asymmetric particle jumps lead to a more general form $p \partial_{x}^{\alpha}+q \partial_{-x}^{\alpha}$ of the fractional derivative operator [7, 4] with symbol $p(-i k)^{\alpha}+q(i k)^{\alpha}$, where $p=1-q$ is the asymptotic fraction of positive jumps as the jump size tends to infinity. For symmetric vector jumps a similar argument leads to $\partial_{t} C=\Delta_{x}^{\alpha / 2} C$ using the fractional Laplacian with symbol $-\|k\|^{\alpha}$; see 14, 16] for more general forms in $\mathbb{R}^{d}$. These pseudodifferential operators are also generators of certain continuous convolution semigroups [2, 11].

If the waiting time distribution between particle jumps is heavy tailed and varies regularly with index $0<\beta<1$ (roughly speaking, the chance of waiting longer than time $t$ before the next jump falls off like $t^{-\beta}$ ), then the random walk of particle jumps (distributed as before) converges to a Lévy motion subordinated to an inverse $\beta$-stable subordinator [17, 18]. Assuming that the waiting time and the ensuing particle jump are independent, the subordinator is independent of the Lévy process, and the governing equation becomes $\partial_{t}^{\beta} C=\partial_{|x|}^{\alpha} C+C(x, 0) t^{-\beta} / \Gamma(1-\beta)$ which was first proposed by Zaslavsky [31] as a model for Hamiltonian chaos. Asymmetric jumps, or vector jumps, modify the spatial derivative in the same manner as before 2]. Heavy tailed particle jumps lead to fractional derivatives in space, and heavy tailed waiting times introduce fractional derivatives in time.

When the waiting times and the particle jumps are dependent random variables, a different form of the governing equation emerges. The limiting process is still a Lévy motion subordinated to an inverse stable subordinator, but now the two processes are dependent. The space-time vector consisting of the waiting time and the jump has to be treated using operator stable limit theory, since each coordinate 
has a different tail behavior [3]. This leads to a governing equation that employs a new kind of coupled space-time fractional derivative. Suppose the waiting time $J$ satisfies $P(J>t)=t^{-\beta}$ for large $t$ and the symmetric particle jump size $Y$ is normally distributed with mean zero and variance $2 t$ when the waiting time $J=t$. Then the governing equation $\left(\partial_{t}-\partial_{x}^{2}\right)^{\beta} C(x, t)=C(x, 0) t^{-\beta} / \Gamma(1-\beta)$ employs a coupled space-time fractional derivative with Fourier-Laplace symbol $\left(s+k^{2}\right)^{\beta}$. The purpose of this paper is to develop the properties of these operators, in order to establish a mathematical basis for the analysis of these equations. What makes this problem interesting is that, since space and time are inexorably linked, one cannot view these evolution equations in the usual manner, as ordinary differential equations on some abstract function space.

\section{Space-time Fractional DeRIVATives}

Let $\mathbb{R}_{+}=[0, \infty)$ and suppose that $\nu(d x, d t)$ is a probability distribution on $\mathbb{R}^{d} \times \mathbb{R}_{+}$with Fourier-Laplace transform

$$
\hat{\nu}(k, s)=\int_{\mathbb{R}^{d} \times \mathbb{R}_{+}} e^{i x \cdot k} e^{-s t} \nu(d x, d t) .
$$

Let $\nu^{n}=\nu * \cdots * \nu$ denote the $n$-fold convolution of $\nu$ with itself. We say that $\nu$ is infinitely divisible if for each $n=1,2,3, \ldots$ there exists a probability distribution $\nu_{n}$ such that $\nu_{n}^{n}=\nu$. The Lévy representation (e.g., see Lemma 2.1 in [3]) states that $\nu$ is infinitely divisible if and only if we can write $\hat{\nu}(k, s)=\exp (\psi(k, s))$ for some unique continuous function $\psi: \mathbb{R}^{d} \times \mathbb{R}_{+} \rightarrow \mathbb{C}$ such that $\psi(0,0)=0, \Re(\psi) \leq 0$ and

$$
\begin{aligned}
\psi(k, s)= & i k \cdot a-b s-\frac{1}{2} k \cdot A k \\
& +\int_{\mathbb{R}^{d} \times \mathbb{R}_{+} \backslash\{(0,0)\}}\left(e^{i k \cdot x} e^{-s t}-1-\frac{i k \cdot x}{1+\|x\|^{2}}\right) \phi(d x, d t)
\end{aligned}
$$

for some uniquely determined $(a, b) \in \mathbb{R}^{d} \times \mathbb{R}_{+}$, some nonnegative definite $d \times d$ matrix $A$, and some positive measure $\phi$ on $\mathbb{R}^{d} \times \mathbb{R}_{+} \backslash\{(0,0)\}$ which is finite on sets bounded away from the origin and which satisfies

$$
\int_{0<\|x\|^{2}+t \leq 1}\left(\|x\|^{2}+t\right) \phi(d x, d t)<\infty .
$$

The measure $\phi$ is called the Lévy measure of $\nu$, and the unique triple $[(a, b), A, \phi]$ is called the Lévy representation of $\nu$. In this case, we define the (possibly fractional) convolution power $\nu^{u}$ to be the infinitely divisible law with Lévy representation $[u a, u A, u \phi]$, so that $\nu^{u}$ has characteristic function $\exp (u \psi(k, s))$ for any $u \geq 0$. Then it follows that $\nu^{u} * \nu^{v}=\nu^{u+v}$ for any $u, v \geq 0$.

Infinitely divisible distributions can be used to define convolution semigroups. Let $L_{\omega}^{1}\left(\mathbb{R}^{d} \times \mathbb{R}_{+}\right)$for $\omega \geq 0$ denote the collection of measurable functions for which the integral and hence the norm

$$
\|f\|_{\omega}:=\int_{0}^{\infty} \int_{\mathbb{R}^{d}} e^{-\omega t}|f(x, t)| d x d t
$$

exists. With this norm, which we will call the $L_{\omega}^{1}$-norm, $L_{\omega}^{1}\left(\mathbb{R}^{d} \times \mathbb{R}_{+}\right)$is a Banach space. Clearly, $L^{1}\left(\mathbb{R}^{d} \times \mathbb{R}_{+}\right) \subset L_{\omega}^{1}\left(\mathbb{R}^{d} \times \mathbb{R}_{+}\right)$with proper containment unless $\omega=0$, in which event the two functions spaces are identical. Further, if $f \in L^{1}\left(\mathbb{R}^{d} \times \mathbb{R}_{+}\right)$, then $\|f\|_{\omega} \leq\|f\|_{1}$. 
A family of bounded linear operators $\{T(t): t \geq 0\}$ on a Banach space $X$, such that $T(0)$ is the identity operator and $T(u+v)=T(u) T(v)$ for all $u, v \geq 0$, is called a semigroup of bounded linear operators on $X$. If $\|T(u) f\| \leq M\|f\|$ for all $f \in X$ and all $u \geq 0$, then the semigroup is uniformly bounded; if in this case $M \leq 1$ we also speak of a contraction semigroup. If $T\left(u_{n}\right) f \rightarrow T(u) f$ in $X$ for all $f \in X$ whenever $u_{n} \rightarrow u$, then the semigroup is strongly continuous. It is easy to check that $\{T(u): u \geq 0\}$ is strongly continuous if $T(u) f \rightarrow f$ in $X$ for all $f \in X$ as $u \downarrow 0$. A semigroup on a Banach lattice is called positive, if $f>0$ implies that $T(u) f \geq 0$ for all $u>0$. A strongly continuous positive contraction semigroup is also called a Feller semigroup. The next result shows that any infinitely divisible law on $\mathbb{R}^{d} \times \mathbb{R}_{+}$defines a Feller semigroup on $L_{\omega}^{1}\left(\mathbb{R}^{d} \times \mathbb{R}_{+}\right)$.

Proposition 3.1. Let $\nu$ be an infinitely divisible law on $\mathbb{R}^{d} \times \mathbb{R}_{+}$and define

$$
T(u) f(x, t)=\int_{0}^{t} \int_{\mathbb{R}^{d}} f(x-y, t-s) \nu^{u}(d y, d s)
$$

for all $f \in L_{\omega}^{1}\left(\mathbb{R}^{d} \times \mathbb{R}_{+}\right)$and all $u \geq 0$. Then the family of linear operators $\{T(u)\}_{u \geq 0}$ has the following properties valid for all $f \in L_{\omega}^{1}\left(\mathbb{R}^{d} \times \mathbb{R}_{+}\right)$:

(a) $T(u+v) f=T(u) T(v) f$ for all $u, v \geq 0$,

(b) $T(0) f=f$,

(c) $f>0$ implies that $T(u) f>0$ for all $u>0$,

(d) $\|T(u) f\|_{\omega} \leq\|f\|_{\omega}$ for all $u \geq 0$,

(e) $\lim _{u \downarrow 0}\|T(u) f-f\|_{\omega}=0$.

Proof. The points (a), (b), and (c) follow immediately from the definition of $\nu$ being infinitely divisible. Point (d) follows from Fubini's theorem:

$$
\begin{aligned}
\|T(u) f\|_{\omega} & =\int_{0}^{\infty} e^{-\omega t} \int_{\mathbb{R}^{d}}\left|\int_{0}^{t} \int_{\mathbb{R}^{d}} f(x-y, t-s) \nu^{u}(d y, d s)\right| d x d t \\
& \leq \int_{0}^{\infty} \int_{\mathbb{R}^{d}} \int_{s}^{\infty} e^{-\omega t} \int_{\mathbb{R}^{d}}|f(x-y, t-s)| d x d t \nu^{u}(d y, d s) \\
& \leq\|f\|_{\omega} .
\end{aligned}
$$

Point (e) is a bit more delicate. We first show that (e) holds for an indicator function on a rectangle, i.e., let $f(x, t)=I_{Q}(x, t)=I((x, t) \in Q)$ where the rectangle $Q=\left\{(x, t) \in \mathbb{R}^{d} \times \mathbb{R}_{+}: a_{0} \leq t \leq b_{0}, a_{i} \leq x_{i} \leq b_{i}\right.$ for $\left.i=1, \ldots, d\right\}$ for some $a, b \in \mathbb{R}_{+} \times \mathbb{R}^{d}$. Then

$$
\begin{aligned}
\int_{0}^{\infty} \int_{\mathbb{R}^{d}}|T(u) f(x, t)| d x d t & =\int_{0}^{\infty} \int_{\mathbb{R}^{d}}\left|\int_{0}^{t} \int_{\mathbb{R}^{d}} f(x-y, t-s) \nu^{u}(d y, d s)\right| d x d t \\
& =\int_{0}^{\infty} \int_{\mathbb{R}^{d}} \iint_{(y, s)+Q} d x d t \nu^{u}(d y, d s) \\
& =\prod_{0 \leq i \leq d}\left(b_{i}-a_{i}\right)
\end{aligned}
$$

for all $u \geq 0$. Since $\nu$ is infinitely divisible we have that $\nu^{u} \Rightarrow \nu^{0}$ as $u \downarrow 0$ (e.g., see Corollary 3.1.4 in [15]), which means that $\nu^{u}(B) \rightarrow \nu^{0}(B)$ for all Borel subsets 
$B \subseteq \mathbb{R}^{d} \times \mathbb{R}_{+}$such that $\nu^{0}(\partial B)=01$ Since $\nu^{0}$ is the point mass at zero, $\nu^{0}(\partial B)=0$ if $(0,0) \notin \partial B$. Then for all $(x, t) \in \mathbb{R}^{d} \times \mathbb{R}_{+}$with $x_{i} \neq a_{i}, b_{i}$ for $i=1, \ldots, d$ and $t \neq a_{0}, b_{0}$, we obtain that

$$
\lim _{u \rightarrow 0^{+}} T(u) f(x, t)=\lim _{u \rightarrow 0^{+}} \nu^{u}((x, t)-Q)=\nu^{0}((x, t)-Q)=f(x, t) .
$$

Since $\nu^{u}$ is a probability measure for all $u$, we can apply the Dominated Convergence Theorem over the finite rectangle $Q$ and obtain

$$
\iint_{Q}|T(u) f(x, t)-f(x, t)| d x d t=\iint_{Q}\left|\nu^{u}((x, t)-Q)-\nu^{0}((x, t)-Q)\right| d x d t \rightarrow 0
$$

and thus, $\iint_{Q} T(u) f(x, t) d x d t \rightarrow \iint f(x, t) d x d t=\prod_{0 \leq i \leq d}\left(b_{i}-a_{i}\right)$. In light of (3.4), this implies that

$$
\iint_{(x, t) \notin Q}|T(u) f(x, t)-f(x, t)| d x d t=\iint_{(x, t) \notin Q}|T(u) f(x, t)| d x d t \rightarrow 0 .
$$

Hence, $\iint|T(u) f(x, t)-f(x, t)| d x d t \rightarrow 0$ so that (e) holds for indicator functions $f$ with the $L^{1}$ norm in place of the $L_{\omega}^{1}$ norm. Since $\|f\|_{\omega} \leq\|f\|_{1}$ whenever $f \in$ $L^{1}\left(\mathbb{R}^{d} \times \mathbb{R}_{+}\right)$, (e) holds for any indicator function $f$. Then it follows easily that (e) holds for any simple function $f=\sum_{j=1}^{n} \alpha_{j} I_{Q_{j}}$ with the $L_{\omega}^{1}$ norm.

Clearly, simple functions are dense in $L_{\omega}^{1}$ for any $\omega \geq 0$, as they are all $L^{1}$ spaces just endowed with a different measure. Now for any $f \in L_{\omega}^{1}\left(\mathbb{R}^{d} \times \mathbb{R}_{+}\right)$there exists $g_{n}=\sum \alpha_{j} I_{Q_{j}}$ with $\left\|g_{n}-f\right\|_{\omega}<n^{-1}$. Using the triangle inequality,

$$
\|T(u) f-f\|_{\omega} \leq\left\|T(u) f-T(u) g_{n}\right\|_{\omega}+\left\|T(u) g_{n}-g_{n}\right\|_{\omega}+\left\|g_{n}-f\right\|_{\omega} .
$$

Now (d) yields $\left\|T(u) f-T(u) g_{n}\right\|_{\omega} \leq\left\|f-g_{n}\right\|_{\omega} \leq n^{-1}$ and thus

$$
\|T(u) f-f\|_{\omega} \leq n^{-1}+\left\|T(u) g_{n}-g_{n}\right\|_{\omega}+n^{-1} .
$$

Since we have shown that

$$
\lim _{u \downarrow 0}\left\|T(u) g_{n}-g_{n}\right\|_{\omega}=0
$$

for any choice of $n$, we have established point (e).

For any strongly continuous semigroup $\{T(u): u>0\}$ on a Banach space $X$ we define the generator

$$
L f=\lim _{u \rightarrow 0^{+}} \frac{T(u) f-f}{u} \text { in } X
$$

meaning that $\left\|u^{-1}(T(u) f-f)-L f\right\| \rightarrow 0$ in the Banach space norm. The domain $D(L)$ of this linear operator is the set of all $f \in X$ for which the limit in (3.7) exists. Then $D(L)$ is dense in $X$, and $L$ is closed, meaning that if $f_{n} \rightarrow f$ and $L f_{n} \rightarrow g$ in $X$, then $f \in D(L)$ and $L f=g$ (see, for example, [23], Cor. I.2.5). In the following theorem we characterize the generator of the semigroup defined in equation (3.3). For any $g \in L_{\omega}^{1}\left(\mathbb{R}^{d} \times \mathbb{R}_{+}\right)$the Fourier-Laplace transform

$$
\hat{g}(k, s):=\int_{\mathbb{R}_{+}} \int_{\mathbb{R}^{d}} e^{i k \cdot x} e^{-s t} g(x, t) d x d t
$$

is defined for all $k \in \mathbb{R}^{d}$ and $s>\omega$.

\footnotetext{
${ }^{1}$ Here $\partial B$ denotes the topological boundary of $B$, i.e. $\partial B=\bar{B} \backslash B^{o}$ where $\bar{B}$ is the closure of $B$, defined as the intersection of all closed sets containing $B$, and $B^{o}$ is the interior of $B$, defined as the union of all open sets contained in $B$.
} 
Theorem 3.2. Suppose that $T$ is defined by equation (3.3) in Proposition 3.1 and set $X=L_{\omega}^{1}\left(\mathbb{R}^{d} \times \mathbb{R}_{+}\right)$. Let $L$ be the generator of this strongly continuous semigroup. Then $\widehat{L f}(k, s)=\psi(k, s) \hat{f}(k, s)$ for all $f \in D(L)$, where $\psi(k, s)$ is given by (3.1), and

$$
D(L)=\{f \in X: \exists h \in X \text { with } \psi(k, s) \hat{f}(k, s)=\hat{h}(k, s)\} .
$$

Furthermore, if $\Sigma$ denotes the subset of $L_{\omega}^{1}\left(\mathbb{R}^{d} \times \mathbb{R}_{+}\right)$whose weak first- and second-order spatial derivatives, as well as weak first order time derivatives, are in $L_{\omega}^{1}\left(\mathbb{R}^{d} \times \mathbb{R}_{+}\right)$, then $\Sigma \subset D(L)$ and for $f \in \Sigma$ we have

$$
\begin{aligned}
L f(x, t) & =-a \cdot \nabla f(x, t)+b \frac{\partial f}{\partial t}(x, t)+\frac{1}{2} \nabla \cdot A \nabla f(x, t) \\
& +\int_{\mathbb{R}^{d} \times \mathbb{R}_{+} \backslash\{(0,0)\}}\left(H(t-s) f(x-y, t-s)-f(x, t)+\frac{\nabla f(x, t) \cdot y}{1+\|y\|^{2}}\right) \phi(d y, d s),
\end{aligned}
$$

where $H(t)$ is the Heaviside step function and $\phi$ is from (3.1).

Proof. If $f \in D(L) \subset L_{\omega}^{1}\left(\mathbb{R}^{d} \times \mathbb{R}_{+}\right)$, then the Fourier-Laplace transform $\hat{f}(k, s)$ exists. Since the Fourier-Laplace transform of a convolution is a product,

$$
\widehat{T(u) f}(k, s)=\exp (u \psi(k, s)) \hat{f}(k, s)
$$

for $(k, s) \in \mathbb{R}^{d} \times(\omega, \infty)$. The Fourier-Laplace transform is a continuous operator in the sense that $\|\hat{f}-\hat{g}\|_{\infty} \leq\|f-g\|_{\omega}$ for any $f, g \in X$. Hence it follows from (3.7) that

$$
\widehat{L f}(k, s)=\lim _{u \rightarrow 0^{+}} \frac{\exp (u \psi(k, s))-1}{u} \hat{f}(k, s)=\psi(k, s) \hat{f}(k, s)
$$

for all $f \in D(L)$ and $(k, s) \in \mathbb{R}^{d} \times(\omega, \infty)$.

Conversely, let $f \in X$ be such that $\psi(k, s) \hat{f}(k, s)=\hat{h}(k, s)$ for some $h \in X$. Then $g:=\lambda f-h \in X$ for all $\lambda>0$. Furthermore, it is a basic fact of semigroup theory (see, e.g., 23, Thm. I.5.2) that the resolvent operator $(\lambda I-L)^{-1}$ is a bounded linear operator for all $\lambda>0$ and maps $X$ into $D(L)$. Let $q=(\lambda I-L)^{-1} g$. Then $\lambda q-L q=g$ and $\lambda \hat{q}(k, s)-\psi(k, s) \hat{q}(k, s)=\hat{g}(k, s)$. Thus

$$
\hat{q}(k, s)=\frac{\hat{g}(k, s)}{\lambda-\psi(k, s)}=\frac{\lambda \hat{f}(k, s)-\psi(k, s) \hat{f}(k, s)}{\lambda-\psi(k, s)}=\hat{f}(k, s) .
$$

Hence $q=f$ and therefore, $f \in D(L)$.

Finally, we establish the form of the generator $L$ in equation (3.8). Let

$$
\|f\|_{\Sigma}=\|f\|_{\omega}+\sum_{i=1}^{d}\left\|\frac{\partial f}{\partial x_{i}}\right\|_{\omega}+\sum_{i, j=1}^{d}\left\|\frac{\partial^{2} f}{\partial x_{i} \partial x_{j}}\right\|_{\omega}+\left\|\frac{\partial f}{\partial t}\right\|_{\omega} .
$$

We assume that $f$ is defined to be zero for $t<0$ and suppress the Heaviside function $H$ written in (3.8). First assume $f$ is continuously differentiable twice in $x$ and once in $t$. Using a Taylor series expansion on $x$,

$$
f(x-y, t)-f(x, t)+y \cdot \nabla f(x, t)=y \cdot \int_{0}^{1}(1-r) M_{x-r y} y d r,
$$

where $M_{x}$ is the Hessian matrix of $f$ evaluated at $(x, t)$. It is easy to check that

$$
\iint e^{-\omega t}\left|f(x-y, t)-f(x, t)+\frac{y \cdot \nabla f(x, t)}{1+\|y\|^{2}}\right| d x d t \leq C\|f\|_{\Sigma} \frac{\|y\|^{2}}{1+\|y\|^{2}}
$$


for some constant $C$. Similarly, using a Taylor series expansion on $t$

$$
\iint e^{-\omega t}|f(x, t-s)-f(x, t)| d x d t \leq D\|f\|_{\Sigma} \frac{s}{1+s}
$$

for some constant $D$.

Using the Fubini theorem - tacitly adding and subtracting $f(x-y, t)$ so we can utilize the preceding estimates - we see that

$$
\begin{aligned}
& \iint e^{-\omega t}\left(\int\left|f(x-y, t-s)-f(x, t)+\frac{y \cdot \nabla f(x, t)}{1+\|y\|^{2}}\right| \phi(d y, d s)\right) d x d t \\
& =\int\left(\iint e^{-\omega t}\left|f(x-y, t-s)-f(x, t)+\frac{y \cdot \nabla f(x, t)}{1+\|y\|^{2}}\right| d x d t\right) \phi(d y, d s) \\
& \leq\|f\|_{\Sigma}\left(C \int \frac{\|y\|^{2}}{1+\|y\|^{2}} \phi(d y, d s)+D \int \frac{s}{1+s} \phi(d y, d s)\right) \leq K\|f\|_{\Sigma}
\end{aligned}
$$

for some constant $K$ in view of (3.2). Thus (3.8) is well defined for all $f$ satisfying the stated regularity conditions.

Since $\left(-i k_{j}\right) \hat{f}(k, s)$ is the Fourier-Laplace transform of $\partial f(x, t) / \partial x_{j}, s \hat{f}(k, s)$ is the (distributional) Fourier-Laplace transform of $\partial f(x, t) / \partial t$ and $f(x-y, t-s)$ has Fourier-Laplace transform $\exp (i k \cdot y) \exp (-s t) \hat{f}(k, s)$, it follows that the right-hand side of 3.8 has Fourier-Laplace transform $\psi(k, s) \hat{f}(k, s)$.

Next we show $\Sigma \subset D(L)$. Let $f \in \Sigma$. First note that if

$$
L_{1} f(x, t):=-a \cdot \nabla f(x, t)-b \frac{\partial f}{\partial t}(x, t)-\frac{1}{2} \nabla \cdot A \nabla f(x, t),
$$

then $\left\|L_{1} f\right\|_{\omega} \leq B\|f\|_{\Sigma}$ for some constant $B$ independent of $f$. Further, note that if

$$
L_{2} f(x, t):=\int_{\mathbb{R}^{d} \times \mathbb{R}_{+} \backslash\{(0,0)\}}\left(f(x-y, t-s)-f(x, t)+\frac{\nabla f(x, t) \cdot y}{1+\|y\|^{2}}\right) \phi(d y, d s),
$$

then $L=L_{1}-L_{2}$ and for $f \in \Sigma \cap C^{\infty}\left(\mathbb{R}^{d} \times \mathbb{R}_{+}\right)$,

$$
\|L f\|_{\omega} \leq\left\|L_{1} f\right\|_{\omega}+\left\|L_{2} f\right\|_{\omega} \leq B\|f\|_{\Sigma}+K\|f\|_{\Sigma} .
$$

Now if $f \in \Sigma$, there is a sequence $\left\{f_{n}\right\}_{n=1}^{\infty} \subset \Sigma \cap C^{\infty}\left(\mathbb{R}^{d} \times \mathbb{R}_{+}\right)$such that $f_{n} \rightarrow f$ in the norm $\|\cdot\|_{\Sigma}$ and hence also in the $L_{\omega}^{1}$ norm. Furthermore, since $\left\{f_{n}\right\}_{n=1}^{\infty}$ is Cauchy in the norm $\|\cdot\|_{\Sigma}$, inequality (3.11) implies that $\left\{L f_{n}\right\}_{n=1}^{\infty}$ is a Cauchy sequence in the norm $\|\cdot\|_{\omega}$ and hence converges to some $g \in L_{\omega}^{1}\left(\mathbb{R}^{d} \times \mathbb{R}_{+}\right)$. Since $L$ is closed, $f \in D(L)$, and $L f=g$.

Remark 3.3. Another consequence of $T(u)$ being a strongly continuous semigroup is that $f_{u}(x, t)=T(u) p(x, t)$ solves the abstract Cauchy problem

$$
\frac{d}{d u} f_{u}=L f_{u} ; \quad f_{0}=p
$$

for $p \in D(L)$. Furthermore, the integrated equation $T(u) p=L \int_{0}^{u} T(v) p d v+p$ holds for all $p \in X$ (see, for example, [23], Theorem I.2.4). This evolution equation must be interpreted with care since now $f_{u}(x, t)$ is a function of both space and time for each $u \geq 0$.

Remark 3.4. While some of the conclusions of Theorem 3.2 can be obtained from the general theory of strongly continuous semigroups [1, [11, 23], we have not seen any results that entail the time variable as part of the state space. As an additional 
difficulty, the function $f_{u}$ in Remark 3.3 does not even have compact support in $\mathbb{R}^{d} \times \mathbb{R}_{+}$for any $u>0$. Also, note that if $p(x, t)$ is a probability density in $x$ for each $t$, then neither $p(x, t)$ nor $f_{u}(x, t)$ has a converging Fourier integral in $(x, t)$.

\section{Coupled SPACE-Time Diffusion EQuAtions}

In this section we apply the results of Section[3in combination with the stochastic process limit theorems in [3] for a coupled continuous time random walk (CTRW). In the CTRW model, each random waiting time $J_{i}>0$ is followed by a random particle jump $Y_{i} \in \mathbb{R}^{d}$. We assume that the space-time vectors $\left(J_{i}, Y_{i}\right)$ are independent for $i=1,2,3, \ldots$, but we allow dependence between $J_{i}$ and $Y_{i}$. If $J_{i}$ has finite mean, a renewal theorem [8] shows that the number of jumps by time $t$ is asymptotically constant, so that the limiting process is the same as for a simple random walk with nonrandom waiting times. On the other hand, if $P(J>t)=t^{-\beta}$ for some $0<\beta<1$ and large $t$, then under mild conditions (essentially, the probability of a large jump also falls off like a power law whose index may vary with coordinate) the random walk of space-time vectors converges to a $d+1$-dimensional operator Lévy motion $\{(D(u), A(u))\}_{u \geq 0}$, where $D(u)$ is a $\beta$-stable subordinator with Laplace transform $e^{-u s^{\beta}}$. More precisely, if $T(n)=\sum_{j=1}^{n} J_{j}$ is the time of the $n$th jump and $S(n)=\sum_{i=1}^{n} Y_{i}$ is the position of the particle after the $n$th jump, then for some invertible linear operators $A_{u}$ on $\mathbb{R}^{d}$ and $b_{u}>0$ we (essentially) assume joint convergence in distribution $\{(B(c) S(c u), b(c) T(c u))\}_{u \geq 0} \Rightarrow\{(A(u), D(u))\}_{u \geq 0}$ as $c \rightarrow \infty$ in a suitable function space. The function $f_{u}(x, t)$ in Remark 3.3 is the probability density of $(A(u), D(u))$ when $L$ is the generator of the associated Feller semigroup. The number of jumps $N_{t}$ by time $t$ is the inverse of the process $\{T(u)\}$ (since $\{T(n) \leq t\}=\left\{N_{t} \geq n\right\}$ ) which leads to $\left\{\tilde{b}(c) N_{c t}\right\} \Rightarrow\{E(t)\}$, where the inverse process $E_{t}=\inf \{u \geq 0: D(u)>t\}$. Finally the normalized CTRW $\left\{\tilde{B}(c) S\left(N_{c t}\right)\right\} \Rightarrow\{A(E(t))\}$, where the limit is a stochastic model for coupled anomalous diffusion at the macroscopic scale $(c \rightarrow \infty)$. A computation in 3] shows that the probability density of this limit process is

$$
h(x, t)=\int_{0}^{\infty} \frac{\partial^{\beta-1}}{\partial t^{\beta-1}} f_{u}(x, t) d u
$$

and the Fourier-Laplace transform of $h$ is simply

$$
\hat{h}(k, s)=\int_{0}^{\infty} s^{\beta-1} e^{u \psi(k, s)} d u=\frac{s^{\beta-1}}{\psi(k, s)}
$$

since $\hat{f}_{u}(k, s)=\exp (u \psi(k, s))$. Then $\psi(k, s) \hat{h}(k, s)=s^{\beta-1}$, where $\psi(k, s)$ is the Fourier-Laplace symbol of the generator $L$. Now suppose that the particle location at time $t=0$ is a random variable $X_{0}$ with $C^{\infty}$ probability density $P(x)$. Under the CTRW model, the random particle location at time $t>0$ is $Z_{t}=X_{0}+A\left(E_{t}\right)$, and assuming $X_{0}$ is independent of everything else, the probability density of $Z_{t}$ is

$$
C(x, t)=\int h(x-y, t) P(y) d y .
$$

Then $\hat{C}(k, s)=\hat{h}(k, s) p(k)$, where $p(k)=\int e^{i k \cdot x} P(x) d x$ is the Fourier transform. Now

$$
\psi(k, s) \hat{C}(k, s)=\psi(k, s) \hat{h}(k, s) p(k)=s^{\beta-1} p(k) .
$$


The right-hand side inverts to $\frac{t^{-\beta}}{\Gamma(1-\beta)} P(x)$, and is thus an element of $L_{\omega}^{1}\left(\mathbb{R}^{d} \times \mathbb{R}_{+}\right)$. Hence $C(x, t)$ satisfies the conditions of Theorem 3.2 so that $C$ is in the domain of $L$ and inverting the above equation leads us to conclude that $C(x, t)$ is the unique Laplace-Fourier transformable solution to the coupled space-time diffusion equation

$$
L C(x, t)=\frac{t^{-\beta}}{\Gamma(1-\beta)} P(x) .
$$

Remark 4.1. The pseudodifferential operator $L$ in (4.3) with Laplace-Fourier symbol $\psi(k, s)$ can be computed using the Lévy representation (3.1) along with Theorem 2.2 in [3], which specifies the form of the Lévy measure $\phi(d x, d t)$. Several examples are contained in [3. In the uncoupled case with symmetric scalar jumps, the symbol $\psi(k, s)=s^{\beta}-|k|^{\alpha}$ corresponds to the uncoupled operator $\partial_{t}^{\beta}-\partial_{|x|}^{\alpha}$, and then (4.3) reduces to the fractional kinetic equation of Zaslavsky 31. If $Y$ is normal mean zero variance $2 t$ when $J=t$, then $\psi(k, s)=\left(s+k^{2}\right)^{\beta}$ and (4.3) becomes

$$
\left(\partial_{t}-\partial_{x}^{2}\right)^{\beta} h(x, t)=P(x) \frac{t^{-\beta}}{\Gamma(1-\beta)} .
$$

Heavy tailed symmetric jumps lead to a similar form with $L=\left(\partial_{t}-\partial_{|x|}^{\alpha}\right)^{\beta}$ in $\mathbb{R}^{1}$ or $L=\left(\partial_{t}-\Delta_{x}^{\alpha / 2}\right)^{\beta}$ in $\mathbb{R}^{d}$.

\section{REFERENCES}

[1] W. Arendt, C. Batty, M. Hieber, and F. Neubrander, Vector-valued Laplace transforms and Cauchy problems. Monographs in Mathematics, Birkhaeuser-Verlag, Berlin (2001). MR 1886588 (2003g:47072)

[2] B. Baeumer and M. Merschaert, Stochastic solutions for fractional Cauchy problems. Frac. Calc. Appl. Anal. 4 (2001), 481-500. MR,1874479 (2003d:26006)

[3] P. Becker-Kern, M.M. Meerschaert and H.P. Scheffler, Limit theorems for coupled continuous time random walks. Ann. Probab. 32 (2004), No. 1B, 730-756. MR2039941 (2004m:60092)

[4] D. Benson, S. Wheatcraft and M. Meerschaert, The fractional-order governing equation of Lévy motion. Water Resources Research 36 (2000), 1413-1424.

[5] A. Blumen, G. Zumofen and J. Klafter, Transport aspects in anomalous diffusion: Lévy walks. Phys Rev. A 40 (1989), 3964-3973.

[6] J.P. Bouchaud and A. Georges, Anomalous diffusion in disordered media - statistical mechanisms, models and physical applications, Phys. Rep. 195 (1990), 127-293. MR.1081295

[7] A. Chaves, A fractional diffusion equation to describe Lévy flights. Phys. Lett. A 239 (1998), 13-16. MF 1616103 (99a:82067)

[8] W. Feller, An Introduction to Probability Theory and Its Applications. Vol. II, 2nd Ed., Wiley, New York (1971). MR0270403 (42:5292)

[9] B. Gnedenko and A. Kolmogorov, Limit Distributions for Sums of Independent Random Variables. Translated from the Russian, annotated, and revised by K. L. Chung. With appendices by J. L. Doob and P. L. Hsu. Revised edition, Addison-Wesley, Reading, Mass (1968). MR0233400 (38:1722)

[10] R. Gorenflo, F. Mainardi, E. Scalas and M. Raberto, Fractional calculus and continuous-time finance. III. The diffusion limit. Mathematical finance (Konstanz, 2000), 171-180, Trends Math., Birkhäuser, Basel (2001). MR 1882830

[11] E. Hille and R.S. Phillips, Functional Analysis and Semi-Groups. Amer. Math. Soc. Coll. Publ. 31, American Mathematical Society, Providence (1957). MR0089373 (19:664d)

[12] Z. Jurek and J.D. Mason, Operator-Limit Distributions in Probability Theory, Wiley, New York (1993). MR 1243181 (95b:60018)

[13] J. Klafter, A. Blumen and M.F. Shlesinger, Stochastic pathways to anomalous diffusion, Phys. Rev. A 35 (1987), 3081-3085. MR0884309 (88d:82132) 
[14] M.M. Meerschaert, D. Benson and B. Bäumer, Multidimensional advection and fractional dispersion. Phys. Rev. E 59 (1999), 5026-5028.

[15] M.M. Meerschaert and H.P. Scheffler, Limit Theorems for Sums of Independent Random Vectors: Heavy Tails in Theory and Practice. Wiley, New York (2001). MR,1840531(2002i:60047)

[16] M.M. Meerschaert, D. Benson and B. Bäumer, Operator Lévy motion and multiscaling anomalous diffusion. Phys. Rev. E 63 (2001), 1112-1117.

[17] M.M. Meerschaert and H.P. Scheffler, Limit theorems for continuous time random walks with infinite mean waiting times. J. Appl. Probab. 41 (2004), No. 2, 455-466. MR2052584

[18] M.M. Meerschaert, D.A. Benson, H.P. Scheffler and B. Baeumer, Stochastic solution of space-time fractional diffusion equations. Phys. Rev. E 65 (2002), 1103-1106. MR 1917983 (2003d:60165)

[19] M.M. Meerschaert, D.A. Benson, H.P. Scheffler and P. Becker-Kern, Governing equations and solutions of anomalous random walk limits. Phys. Rev. E 66 (2002), 102-105.

[20] Metzler R. and J. Klafter, The random walk's guide to anomalous diffusion: A fractional dynamics approach, Phys. Rep. 339 (2000), 1-77. MR1809268 (2001k:82082)

[21] K.S. Miller and B. Ross, An introduction to the fractional calculus and fractional differential equations. Wiley, New York (1993). MR1219954 (94e:26013)

[22] E.W. Montroll and G.H. Weiss, Random walks on lattices. II. J. Mathematical Phys. 6 (1965), 167-181. MR0172344 (30:2563)

[23] A. Pazy, Semigroups of Linear Operators and Applications to Partial Differential equations. Applied Mathematical Sciences 44, Springer-Verlag, New York (1983). MR0710486 (85g:47061)

[24] M. Raberto, E. Scalas and F. Mainardi, Waiting-times and returns in high-frequency financial data: an empirical study. Physica A 314 (2002), 749-755.

[25] L. Sabatelli, S. Keating, J. Dudley, P. Richmond, Waiting time distributions in financial markets. Eur. Phys. J B 27 (2002), 273-275. MR.1930869

[26] A.I. Saichev and G.M. Zaslavsky, Fractional kinetic equations: Solutions and applications. Chaos 7 (1997), 753-764. MR:1604710 (98k:82162)

[27] S. Samko, A. Kilbas and O. Marichev, Fractional Integrals and derivatives: Theory and Applications. Gordon and Breach, London (1993). MR.1347689 (96d:26012)

[28] E. Scalas, R. Gorenflo and F. Mainardi, Fractional calculus and continuous-time finance. Phys. A 284 (2000), 376-384. MR1773804 (2001d:91104)

[29] H. Scher and M. Lax, Stochastic transport in a disordered solid. I. Theory. Phys. Rev. B 7 (1973), 4491-4502. MR0391854 (52:12673)

[30] V.V. Uchaikin and V.M. Zolotarev, Chance and stability. Stable distributions and their applications. VSP, Utrecht (1999). MF1745764 (2002a:60002)

[31] G. Zaslavsky, Fractional kinetic equation for Hamiltonian chaos. Chaotic advection, tracer dynamics and turbulent dispersion. Phys. D 76 (1994), 110-122. MR1295881 (95h:58120)

Department of Mathematics \& Statistics, University of Otago, Dunedin, New Zealand

E-mail address: bbaeumer@maths.otago.ac.nz

Department of Physics, University of Nevada, Reno, Nevada 89557-0084

E-mail address: mcubed@unr.edu

Current address: Department of Mathematics \& Statistics, University of Otago, Dunedin, New Zealand

E-mail address: mcubed@maths.otago.ac.nz

Department of Mathematics, University of Nevada, Reno, Nevada 89557-0084

E-mail address: jm@unr.edu 